\title{
Resonance fluorescence from an atomic-quantum-memory compatible single photon source based on GaAs droplet quantum dots
}

\author{
Laxmi Narayan Tripathi, ${ }^{1,2}$ Yu-Ming He, ${ }^{3,4}$ Łukasz Dusanowski, ${ }^{1}$ Piotr Andrzej Wroński, ${ }^{1}$ Chao-Yang Lu, ${ }^{3,4}$ \\ Christian Schneider, ${ }^{1}$ and Sven Höfling ${ }^{1,3,5}$, a) \\ 1) Technische Physik and Wilhelm-Conrad-Röntgen Research Center for Complex Material Systems, \\ Universität Würzburg, Am Hubland, Würzburg-970\%4, Germany \\ ${ }^{2)}$ Department of Physics, Birla Institute of Technology, Mesra, Ranchi 835215, Jharkhand, \\ India \\ ${ }^{3)}$ National Laboratory for Physical Sciences at Microscale and Department of Modern Physics, \\ University of Science and Technology of China, Shanghai Branch, Shanghai 201315, \\ China \\ ${ }^{4)}$ CAS-Alibaba Quantum Computing Laboratory, CAS Centre for Excellence in Quantum Information \\ and Quantum Physics, University of Science and Technology of China, Shanghai 201315, \\ China \\ ${ }^{5)}$ SUPA, School of Physics and Astronomy, University of St. Andrews, St. Andrews KY16 9SS, \\ United Kingdom
}

(Dated: June 19, 2018)

Single photon sources, which are compatible with quantum memories are an important component of quantum networks. In this article, we show optical investigations on isolated $\mathrm{GaAs} / \mathrm{Al}_{0.25} \mathrm{Ga}_{0.75}$ As quantum dots grown via droplet epitaxy, which emit single photons on resonance with the Rb-87- $\mathrm{D}_{2}$ line $(780 \mathrm{~nm})$. Under continuous wave resonant excitation conditions, we observe bright, clean and narrowband resonance fluorescence emission from such a droplet quantum dot. Furthermore, the second-order correlation measurement clearly demonstrates the single photon emission from this resonantly driven transition. Spectrally resolved resonance fluorescence of a similar quantum dot yields a linewidth as narrow as $660 \mathrm{MHz}(2.7 \mu \mathrm{eV})$, which corresponds to a coherence time of $0.482 \mathrm{~ns}$. The observed linewidth is the smallest reported so far for strain free GaAs quantum dots grown via the droplet method. We believe that this single photon source can be a prime candidate for applications in optical quantum networks.

PACS numbers: Valid PACS appear here

Keywords: Single photon source, quantum memory, droplet quantum dots, Resonance Florescence, droplet epitaxy

Long distance quantum communication based on quantum repeater schemes ${ }^{1}$ can be realized by a combination of high-performance single photon sources ${ }^{2}$ and suitable quantum memories. ${ }^{3-6}$ For this application single photon sources should possess not only high photon fluxes and very narrow linewidth, but also match spectrally with other components of the system ${ }^{7}$ such as long-lived quantum memories ${ }^{8}$ and which may be utilized in building blocks of quantum repeater nodes. ${ }^{9,10}$ Semiconductor quantum dots (QDs) have been shown to be good candidates for efficient and highly indistinguishable single photon sources. ${ }^{11-13}$ Moreover, first prototypes of a hybrid semiconductor QD-atomic interface ${ }^{14}$ have been already realized to demonstrate the principle feasibility of this approach. Coupling of atomic clouds of Rubidium $(\mathrm{Rb})$ with QDs allowed for demonstration of compact, tunable and spectrally selective delay lines for single photons. ${ }^{15}$ Furthermore, the emission wavelength of solid-state GaAs/AlGaAs QDs were tuned in the spectral range of $\mathrm{Rb}-87-\mathrm{D}_{2}$ lines $(780 \mathrm{~nm})$ by introducing strain $^{16,17}$ and even operated under electrical pumping. ${ }^{18}$

\footnotetext{
a) Electronic mail: Sven.Hoefling@physik.uni-wuerzburg.de
}

A crucial resource for applications of single photons in quantum networks ${ }^{19}$ and linear optical computing, ${ }^{20,21}$ are high degrees of indistinguishably of the emitted photons. The photons should be identical in color, polarization and their coherence should be Fourier limited. ${ }^{22}$ However, the quality of single photons emitted from the semiconductor QDs critically depends on excitation conditions. Highest degrees of indistinguishability of single photons have been observed so far under resonant excitation conditions ${ }^{11-13}$ as opposed to non-resonant excitation. Furthermore, continuous wave $(\mathrm{CW})$ resonance fluorescence provided a paradigm approach to demonstrate high degrees of indistinguishability and measure single QD coherence time by probing linewidth of a QD transition, reaching close to lifetime limited values. ${ }^{23,24}$

In this work, we focus on a resonance fluorescence measurements of single $\mathrm{GaAs} / \mathrm{Al}_{0.25} \mathrm{Ga}_{0.75} \mathrm{As}$ QDs grown by droplet epitaxy. We show that the energy of emitted single photons from selected QDs match the Rb- $87-\mathrm{D}_{2}$ lines $(780 \mathrm{~nm}){ }^{25}$ Furthermore, the resonance fluorescence measurements yield a spectral linewidth of only 660 $\mathrm{MHz}$ corresponding to a coherence time $\left(\tau_{c o h}\right)$ of 0.482 ns ( using the relation, linewidth $=\frac{1}{\pi \tau_{c o h}}$ ). These measurements strongly outline the feasibility to implement droplet epitaxy grown QDs as single photon sources in 
quantum information schemes.

Our sample consists of a low-density layer of GaAs $/ \mathrm{Al}_{0.25} \mathrm{Ga}_{0.75} \mathrm{As}$ QDs grown via droplet epitaxy. The layer of the QDs is embedded in a Schottky-diode structure. The sample was grown by solid-source molecular beam epitaxy (MBE) on a GaAs(100) semi-insulating a substrate. ${ }^{26,27} \mathrm{Ga}$ and $\mathrm{Al}$ were supplied by standard effusion cells while $\mathrm{As}_{4}$ molecules were provided by a valved cracker source. After the growth of a GaAs buffer layer, a $50 \mathrm{~nm}$ Si-doped GaAs layers and a $150 \mathrm{~nm}$ thick, Sidoped $\mathrm{Al}_{0.25} \mathrm{Ga}_{0.75}$ As layer were deposited on the substrate at the temperature of $550{ }^{\circ} \mathrm{C}$. The doping concentration was kept constant to be $3 \times 10^{18} \mathrm{~cm} .^{-3}$ Further, a $20 \mathrm{~nm}$ undoped $\mathrm{Al}_{0.25} \mathrm{Ga}_{0.75}$ As tunnel barrier was grown below the QDs at substrate temperature of $350{ }^{\circ} \mathrm{C}$ and the As valve was closed to reduce the background As pressure. Moreover, Ga was deposited at a flow rate of 0.071 monolayer/s for 2 minutes and crystallized with As at 3 $\times 10^{-5}$ Torr beam equivalent pressure. Followed by a rise of substrate temperature to $400{ }^{\circ} \mathrm{C}$, the GaAs QDs were in-situ annealed for $10 \mathrm{~min}$ to cure crystal defects. Next, the growth was continued with the AlGaAs QD capping layer. The overgrowth of QDs with $10 \mathrm{~nm} \mathrm{Al} \mathrm{Al}_{0.25} \mathrm{Ga}$ ${ }_{0.75}$ As was done by migration enhanced epitaxy, ${ }^{27}$ followed by raising the substrate temperature to $550{ }^{\circ} \mathrm{C}$ and 10 periods of a $2 \mathrm{~nm} \mathrm{AlAs} / 2 \mathrm{~nm} \mathrm{Al}_{0.25} \mathrm{Ga}_{0.75} \mathrm{As}$ superlattice. The sample growth was completed with 60 $\mathrm{nm} \mathrm{Al}_{0.25} \mathrm{Ga}_{0.75} \mathrm{As}$ and a $15 \mathrm{~nm}$ GaAs cap layer. The areal density of the QDs was found to be $5 \times 10^{9} \mathrm{~cm}^{-2}$.

For $\mu$ photoluminescence (PL) and resonance fluorescence $(\mathrm{RF})$ measurements the sample was kept in a closed-cycle cryostat at $5 \mathrm{~K}$. Non-resonant $\mu \mathrm{PL}$ measurements were done using CW $532 \mathrm{~nm}$ laser, while resonantly excited by a $780.076 \mathrm{~nm}$ laser. The emitted light from the sample was collected into a spectrometer (1200 lines, grating resolution $0.01 \mathrm{~nm}$ ) by a $50 \mathrm{X}$ objective $(\mathrm{NA}=0.42)$.

Figure $1 \mathrm{~b}$ depicts a photoluminescence (PL) spectrum of a droplet GaAs $/ \mathrm{Al}_{0.25} \mathrm{Ga}_{0.75} \mathrm{As}$ QDs, which was excited under non-resonant excitation $(532 \mathrm{~nm})$. Apart from a distinct QD line at $780 \mathrm{~nm}$, we observe several other QD-attributed emission features, which most likely stem from other QDs excited within laser spot size. The diameter of the laser spot is $\sim 0.9 \mu \mathrm{m}$ for $532 \mathrm{~nm}$ and we used 50X magnification objective. Considering the areal density of QDs which is $\sim 5 \times 10^{9} \mathrm{~cm},{ }^{-2}$ we estimate that about 35 quantum dots might be excited. In order to single out the emission line of interest, we resonantly drive the QD with the fundamental transition at $780 \mathrm{~nm}$ with a narrow-band $\mathrm{CW}$ laser. As shown in the spectrum in Fig. 1(c), this method clearly yields a purely monochromatic emission by selectively driving the ground state of the QD. The excitation scheme and energy levels for a resonant excitation is shown in Fig. 1(a).

More importantly, the resonance fluorescence spectrum shows that the emitter of interest can be coherently driven at a frequency which overlaps with the Rb- $87-\mathrm{D}_{2}$ lines $(780 \mathrm{~nm})$. Further, it is also seen that the emitter (a)

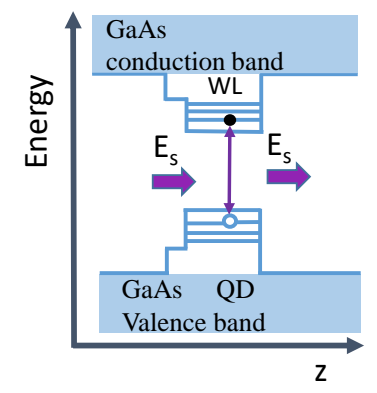

(b)

(c)

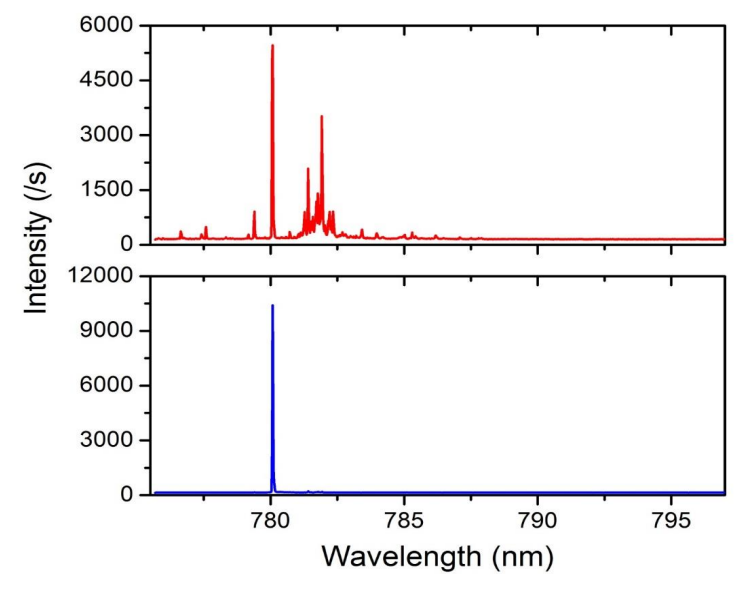

Figure 1. a) A schematic drawing for the resonant excitation $\left(E_{s}\right)$ scheme of the QD. b) Photoluminescence spectrum of a droplet GaAs $/ \mathrm{Al}_{0.25} \mathrm{Ga}_{0.75}$ As QD under non-resonant excitation at $532 \mathrm{~nm}$ with excitation power of $2 \mu \mathrm{W}$. c) Resonant photoluminescence spectrum, yielding a single transition line at $780 \mathrm{~nm}$.

under resonance fluorescence is twice brighter than the one excited under non-resonant scheme under similar excitation power $(2 \mu \mathrm{W})$.

Our method to acquire a pure, nearly background-free resonant emission spectrum from a solid-state quantum emitter is shown in Fig. 2(a). The driving laser is polarized by a combination of a linear polarizer and a halfwave plate, and is focused on the sample surface by a high NA objective. The resonance fluorescence signal from the QD is then collected by the same objective. A second perpendicular linear polarizer filters out the laser signal. Spatial filtering is achieved by focusing the collected RF signal into a single mode fiber, before the signal is dispersed in a monochromator and recorded on a CCD camera. In order to accurately deduce the linewidth of our droplet QDs, we performed resonant laser scans under CW excitation [Fig. 2(b)]. RF counts were recorded as a function of the laser frequency. There, we chose a pump power of $0.1 \mu \mathrm{W}$, well below the saturation of the QD transition and an integration time of $20 \mathrm{~s}$. Two resonance fluorescence peaks evolve in the spectrum, which correspond to the fine structure splitting (FSS) of 1.25 $\mathrm{GHz}$ of the QD emitter. The FSS is relatively small, due to a greater degree of structural symmetry ${ }^{28,29}$ in droplet quantum dots than a Stranski-Krastanov QD. ${ }^{30-32}$ For example, Huo et al. ${ }^{30}$ reported FSS values ranging from 
(a)

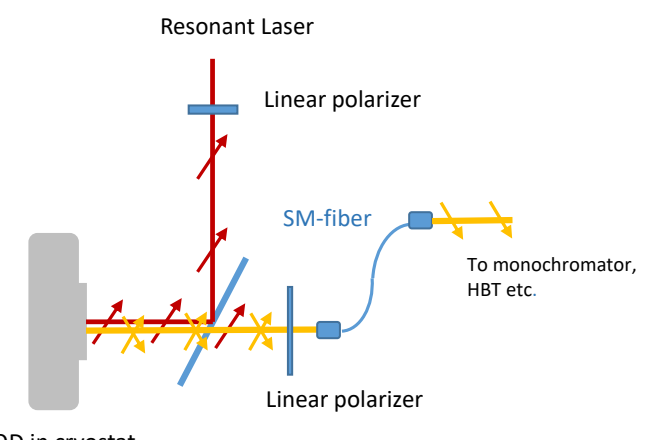

QD in cryostat

(b)

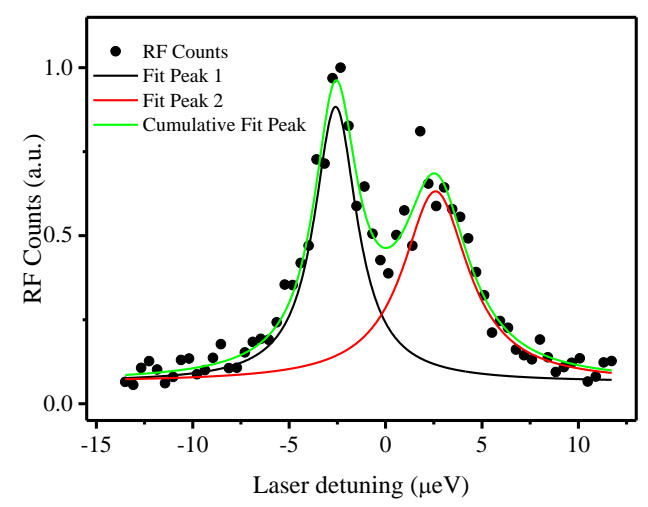

Figure 2. a) A scheme of cross-polarization resonance fluorescence setup. A laser beam (CW $780.076 \mathrm{~nm}$, resonant to the ground state transition of the QD is passed through a linear polarizer and a microscope objective to excite the QD. The laser beam reflected from the sample surface is then filtered by a second linear polarizer oriented perpendicular to the first one. The resonance fluorescence signal emitted from the QD is then collected by the same objective and coupled to a single mode (SM) fiber working as a spatial filter. Finally the optical signal is introduced to the monochromator equipped with a charged coupled camera detector and Hanbury Brown-Twiss (HBT) interferometer. b) The resonance fluorescence spectrum recorded by a laser scan. Solid lines present Lorentzian fits showing two peaks originating from the same QD with a fine structure splitting of about $1.25 \mathrm{GHz}$. The peak 1 has a linewidth of $(0.66 \pm 0.05) \mathrm{GHz}$ and peak 2 has a linewidth of $(1.00 \pm 0.09) \mathrm{GHz}$. The signal to noise ratio is $\sim 11: 1$.

\section{$2.4 \mathrm{GHz}$ to $0.85 \mathrm{GHz}$.}

In order to confirm the quantum nature of our narrowband emission feature, we performed power dependent measurements shown in Fig. 3(a). The excitation power dependent PL intensity (Fig. 3a) is fitted with the equation for a two-level system, ${ }^{33}$

$$
I=I_{\text {sat }}\left(1 /\left(1+P_{n} / P_{\text {exc }}\right)\right),
$$

where, $I_{\text {sat }}$ is the saturation intensity, $P_{\text {exc }}$ is the excitation power and $P_{n}$ is the normalization power. A second-order correlation function for a two-level system is given by ${ }^{34}$

$$
g^{2}(\tau)=1-a \times e^{-\frac{\left|\tau-\tau_{0}\right|}{\tau_{c_{1}}}}
$$

(a)
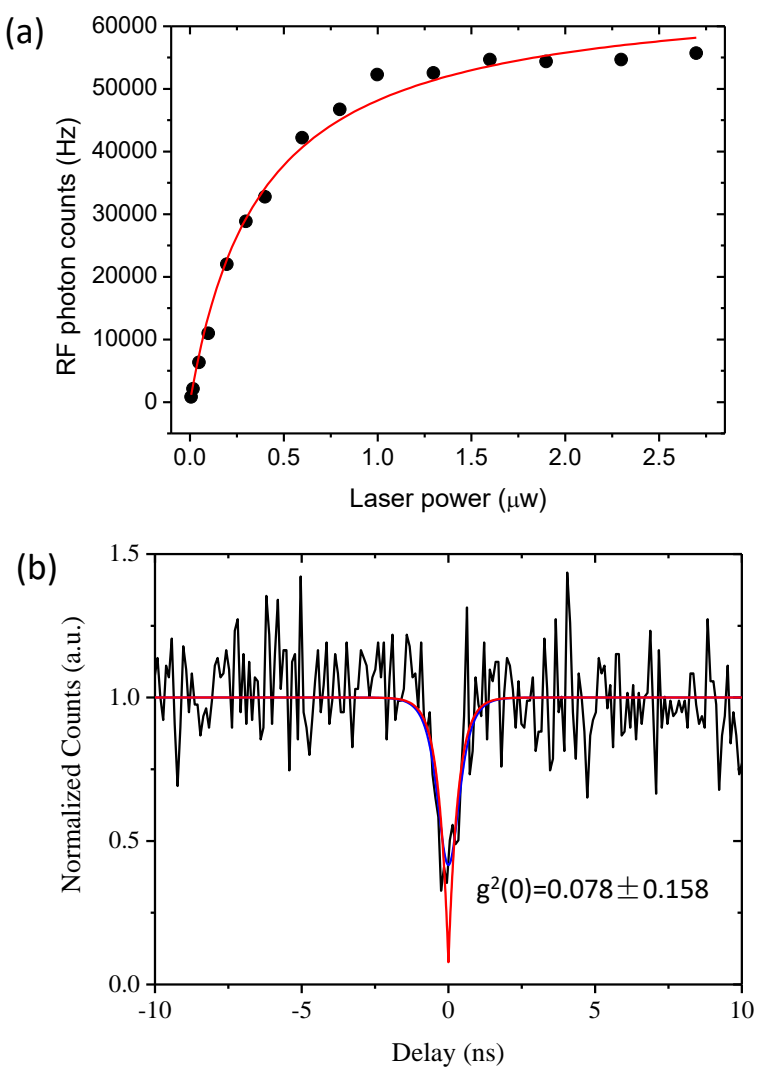

Figure 3. (a) Resonance fluorescence photon (RF) counts as a function of increasing power of the excitation CW laser. The red line is a fit (using Eq. 1), showing a saturation of emission intensity with increase in excitation power. The best-fit normalizations power, $P_{n}=0.376 \mu W$. b) Second order correlation function $g^{2}(\tau)$ as a function of time delay between emitted photons. The blue line is the fit to the intensity autocorrelation measurement data without deconvolution. The red line shows deconvolution fit. The best-fit second-order correlation function $g^{2}(0)$ at zero time delay was found to be $0.078 \pm 0.158$.

here, $\tau_{c_{1}}$ accounts for the exciton relaxation and decay rates of a two-level system and a is a fitting parameter. $\tau, \tau_{0}$ are the time delay and the time delay offset, respectively.

The finite-time resolution of our single-photon detector (350 ps) is accounted for by introducing a Gaussian response function $G_{d}$ given by ${ }^{34}$

$$
G_{d}=\frac{1}{\sigma \sqrt{\pi}} e^{-\frac{1}{2}\left(\frac{\tau-\tau_{0}}{\sigma}\right)^{2}} .
$$

Here, $2 \sqrt{2 \ln (2 \sigma)}$ represent 350 ps timing resolution of our avalanche photo diode. Hence, the convoluted fitting function, $g_{\text {real }}^{2}(\tau)$ is given by

$$
g_{\text {real }}^{2}(\tau)=G_{d} \otimes g^{2}(\tau) .
$$

Here, $\otimes$ represent the convolution operator. In Fig. 3 (b) $g_{\text {real }}^{2}(\tau)$ was used to fit the data to obtain the value of 
the second-order correlation function at zero delay, $g^{2}(0)$ to be $0.078 \pm 0.158$ as shown in Fig. 3(b). The nonzero $g^{2}(0)$ could be due to presence of a non-filtered laser background.

The Schottky diode geometry used in our study enables us to charge tune the emission and spectrally fine tune the emission line of quantized energy levels of the $\mathrm{QDs}^{27}$. We observed resonance fluorescence in both contacted and non-electrically-contacted geometry, and about 50 $\%$ of the investigated QDs showed resonance fluorescence. We note that in contrast to investigations on high quality InGaAs quantum dots in high quality micropillar cavities, ${ }^{11,36}$ the application of additional weak $532 \mathrm{~nm}$ laser light on the investigated GaAs/AlGaAs turned out to improve the QD properties similar to other studies, for instance, Gazzano et al. ${ }^{35}$ The measured linewidths of the doublets in the RF spectrum $(660 \mathrm{MHz}$ and $1 \mathrm{GHz})$ are larger than the Fourier-transform limit of $488 \mathrm{MHz}$ for the exciton relaxation time of $330 \mathrm{ps}$. This difference can be attributed to an inhomogeneous broadening due to charge noise via the d.c. Stark shift. ${ }^{37}$

In summary, we report a solid state semiconductor single photon device based on isolated charge-tunable droplet $\mathrm{Al}_{0.25} \mathrm{Ga}_{0.75} \mathrm{As}$ QDs. Our resonance fluorescence measurement yield important information about linewidth and fine structure splitting. Most importantly the $\mathrm{QD}$ is shown to emit single photons at $780 \mathrm{~nm}$ in resonance with $\mathrm{Rb}-87-\mathrm{D}_{2}$ lines $(780 \mathrm{~nm})$. Continuous wave laser power dependent fluorescence measurement demonstrate two-level behavior of probed emitter. We suggest, that the QD device may find application in quantum communication devices.

This project has received funding from the European Union's Horizon 2020 research and innovation programme under the Marie Skłodowska-Curie grant agreement No 721394. We furthermore gratefully acknowledge support by the State of Bavaria.

\section{REFERENCES}

${ }^{1}$ N. Sangouard, C. Simon, J. Minar, H. Zbinden, H. de Riedmatten, and N. Gisin, Phys. Rev. A 76, 050301 (2007).

${ }^{2}$ I. Aharonovich, D. Englund, and M. Toth, Nat. Photonics 10, 631 (2016), wOS:000384951900008.

${ }^{3}$ A. I. Lvovsky, B. C. Sanders, and W. Tittel, Nat. Photonics 3, 706 (2009).

${ }^{4}$ M. Fleischhauer and M. D. Lukin, Phys. Rev. A 65, 022314 (2002).

${ }^{5}$ X. Maitre, E. Hagley, G. Nogues, C. Wunderlich, P. Goy, M. Brune, J. M. Raimond, and S. Haroche, Phys. Rev. Lett. 79, 769 (1997).

${ }^{6}$ C. Simon, M. Afzelius, J. Appel, A. B. de la Giroday, S. J. Dewhurst, N. Gisin, C. Y. Hu, F. Jelezko, S. Kroll, J. H. Muller, J. Nunn, E. S. Polzik, J. G. Rarity, H. De Riedmatten, W. Rosenfeld, A. J. Shields, N. Skold, R. M. Stevenson, R. Thew, I. A. Walmsley, M. C. Weber, H. Weinfurter, J. Wrachtrup, and R. J. Young, Eur. Phys. J. D 58, 1 (2010).

${ }^{7}$ P. Siyushev, G. Stein, J. Wrachtrup, and I. Gerhardt, Nature 509, 66 (2014).

${ }^{8}$ K. Heshami, D. G. England, P. C. Humphreys, P. J. Bustard,
V. M. Acosta, J. Nunn, and B. J. Sussman, J. Mod. Opt. 63 2005 (2016).

${ }^{9}$ M. T. Rakher, R. J. Warburton, and P. Treutlein, Phys. Rev. A 88, 053834 (2013).

${ }^{10}$ H. J. Briegel, W. Dur, J. I. Cirac, and P. Zoller, Phys. Rev. Lett. 81, 5932 (1998).

${ }^{11}$ X. Ding, Y. He, Z.-C. Duan, N. Gregersen, M.-C. Chen, S. Unsleber, S. Maier, C. Schneider, M. Kamp, S. Hö̈ling, C.-Y. Lu, and J.-W. Pan, Phys. Rev. Lett. 116, 020401 (2016).

${ }^{12}$ N. Somaschi, V. Giesz, L. D. Santis, J. C. Loredo, M. P. Almeida, G. Hornecker, S. L. Portalupi, T. Grange, C. Antón, J. Demory, C. Gómez, I. Sagnes, N. D. Lanzillotti-Kimura, A. Lemaítre, A. Auffeves, A. G. White, L. Lanco, and P. Senellart, Nat. Photonics 10, 340 (2016).

${ }^{13}$ S. Unsleber, Y.-M. He, S. Gerhardt, S. Maier, C.-Y. Lu, J.-W. Pan, N. Gregersen, M. Kamp, C. Schneider, and S. Höfling, Opt. Express 24, 8539 (2016).

${ }^{14}$ N. Akopian, L. Wang, A. Rastelli, O. G. Schmidt, and V. Zwiller, Nat. Photonics 5, 230 (2011).

${ }^{15}$ J. S. Wildmann, R. Trotta, J. Martin-Sanchez, E. Zallo, M. O'Steen, O. G. Schmidt, and A. Rastelli, Phys. Rev. B 92, 235306 (2015).

${ }^{16}$ S. Kumar, R. Trotta, E. Zallo, J. D. Plumhof, P. Atkinson, A. Rastelli, and O. G. Schmidt, Appl. Phys. Lett. 99, 161118 (2011).

${ }^{17}$ R. Trotta, J. Martin-Sanchez, J. S. Wildmann, G. Piredda, M. Reindl, C. Schimpf, E. Zallo, S. Stroj, J. Edlinger, and A. Rastelli, Nat. Commun. 7, 10375 (2016).

${ }^{18}$ H. Huang, R. Trotta, Y. Huo, T. Lettner, J. S. Wildmann, J. Martín-Sánchez, D. Huber, M. Reindl, J. Zhang, E. Zallo, O. G. Schmidt, and A. Rastelli, ACS Photonics 4, 868 (2017).

${ }^{19}$ P. Kok, W. J. Munro, K. Nemoto, T. C. Ralph, J. P. Dowling, and G. J. Milburn, Rev. Mod. Phys. 79, 135 (2007).

${ }^{20}$ J. L. O'Brien, Science 318, 1567 (2007).

${ }^{21}$ J.-W. Pan, Z.-B. Chen, C.-Y. Lu, H. Weinfurter, A. Zeilinger, and M. Żukowski, Rev. Mod. Phys. 84, 777 (2012).

${ }^{22}$ C. Schneider, P. Gold, C.-Y. Lu, S. Höfling, J.-W. Pan, and M. Kamp, in Engineering the Atom-Photon Interaction (Springer, 2015) pp. 343-361.

${ }^{23}$ C. Matthiesen, A. N. Vamivakas, and M. Atatuere, Phys. Rev. Lett. 108, 093602 (2012).

${ }^{24}$ S. Ates, S. M. Ulrich, A. Ulhaq, S. Reitzenstein, A. Loffler, S. Hofling, A. Forchel, and P. Michler, Nat. Photonics 3, 724 (2009).

${ }^{25}$ P. Siddons, C. S. Adams, C. Ge, and I. G. Hughes, Journal of Physics B-atomic Molecular and Optical Physics 41, 155004 (2008).

${ }^{26}$ K. Watanabe, N. Koguchi, and Y. Gotoh, Japanese Journal of Applied Physics Part 2-letters 39, L79 (2000).

${ }^{27}$ F. Langer, D. Plischke, M. Kamp, and S. Höfling, Appl. Phys. Lett. 105, 081111 (2014).

${ }^{28}$ M. Abbarchi, C. A. Mastrandrea, T. Kuroda, T. Mano, K. Sakoda, N. Koguchi, S. Sanguinetti, A. Vinattieri, and M. Gurioli, Phys. Rev. B 78, 125321 (2008).

${ }^{29}$ T. Mano, M. Abbarchi, T. Kuroda, B. McSkimming, A. Ohtake, K. Mitsuishi, and K. Sakoda, Appl. Phys Express 3, 065203 (2010).

${ }^{30}$ Y. Huo, A. Rastelli, and O. Schmidt, Appl. Phys. Lett. 102, 152105 (2013).

${ }^{31}$ D. Huber, M. Reindl, Y. Huo, H. Huang, J. S. Wildmann, O. G. Schmidt, A. Rastelli, and R. Trotta, Nat. Commun. 8, 15506 (2017).

${ }^{32}$ F. Basso Basset, S. Bietti, M. Reindl, L. Esposito, A. Fedorov, D. Huber, A. Rastelli, E. Bonera, R. Trotta, and S. Sanguinetti, Nano Lett. 18, 505 (2018).

${ }^{33}$ S. Kumar, A. Kaczmarczyk, and B. D. Gerardot, Nano Letters, Nano Lett. 15, 7567 (2015).

${ }^{34}$ Y.-M. He, O. Iff, N. Lundt, V. Baumann, M. Davanco, K. Srinivasan, S. Höfling, and C. Schneider, Nat. Commun. 7, 13409 (2016). 
${ }^{35}$ O. Gazzano, S. Michaelis de Vasconcellos, C. Arnold, A. Nowak, E. Galopin, I. Sagnes, L. Lanco, A. Lemaître, and P. Senellart, Nat. Commun. 4, 1425 (2013).

${ }^{36}$ H. Wang, Z.-C. Duan, Y.-H. Li, S. Chen, J.-P. Li, Y.-M. He, M.-
C. Chen, Y. He, X. Ding, C.-Z. Peng, C. Schneider, M. Kamp, S. Höfling, C.-Y. Lu, and J.-W. Pan, Phys. Rev. Lett. 116, 213601 (2016).

${ }^{37}$ A. V. Kuhlmann, J. H. Prechtel, J. Houel, A. Ludwig, D. Reuter, A. D. Wieck, and R. J. Warburton, Nat. Commun. 6, (2015). 